\title{
Pins homolog LGN regulates meiotic spindle organization in mouse oocytes
}

\author{
Xinzheng Guo ${ }^{1,2}$, Shaorong Gao ${ }^{2}$ \\ ${ }^{1}$ College of Life Science, Peking University, Beijing 100871, China; ${ }^{2}$ National Institute of Biological Sciences, Beijing 102206, \\ China
}

Mouse oocytes undergo polarization during meiotic maturation, and this polarization is essential for asymmetric cell divisions that maximize retention of maternal components required for early development. Without conventional centrosomes, the meiotic spindle has less focused poles and is barrel-shaped. The migration of meiotic spindles to the cortex is accompanied by a local reorganization and polarization of the cortex. LGN is a conserved protein involved in cell polarity and regulation of spindle organization. In the present study, we characterized the localization dynamics of LGN during mouse oocyte maturation and analyzed the effects of LGN upregulation and downregulation on meiotic spindle organization. At the germinal vesicle stage, LGN is distributed both cytoplasmically and at the cortex. During maturation, LGN localizes to the meiotic spindle apparatus and cortical LGN becomes less concentrated at the actin cap region. Excessive LGN induces meiotic spindle organization defects by elongating the spindle and enhancing pole focusing, whereas depletion of LGN by RNA interference results in meiotic spindle deformation and chromosome misalignment. Furthermore, the N-terminus of LGN has the ability of full-length LGN to regulate spindle organization, whereas the C-terminus of LGN controls cortical localization and polarization. Our results reveal that LGN is cortically polarized in mouse oocytes and is critical for meiotic spindle organization.

Keywords: LGN, oocyte, cortex, polarization, meiotic spindle organization, $\gamma$-tubulin

Cell Research (2009) 19:838-848. doi: 10.1038/cr.2009.54; published online 12 May 2009

\section{Introduction}

Mouse oocytes become polarized during maturation and undergo typical asymmetric cell divisions to extrude polar bodies [1]. Immature oocytes have a centrally located germinal vesicle (GV) and exhibit no apparent polarity. When meiosis resumes, GV breakdown (GVBD) occurs and then chromatin is condensed to chromosomes, with microtubules polymerizing around. Typical metaphase I (MI) spindles organize without conventional centrosomes. Different from spindles of somatic cells, which have astral microtubules and are diamondshaped, the spindles of mouse oocytes have no astral microtubules and are barrel-shaped [2,3]. Although the mechanism is not well defined, MI spindles migrate to

Correspondence: Shaorong Gao

Tel: +86-10-80728967; Fax: +86-10-80727535

E-mail: gaoshaorong@nibs.ac.cn

Received 16 October 2008; revised 29 December 2008; accepted 20

January 2009; published online 12 May 2009 the nearest part of the cortex. At the same time, prominent cortical polarity develops. At the cortical region overlying the coming spindle, local reorganization occurs. Microfilaments are enriched and form a local actinrich region called the actin cap. When the spindle reaches the cortex, the metaphase to anaphase transition is triggered and the first polar body is extruded. Subsequently, metaphase II (MII) spindles form and are located under the actin cap with the long axis approximately parallel to the cortex $[1,4]$. The molecular details of oocyte cortical polarization and meiotic spindle organization are only beginning to emerge.

Recently, it has been reported that the small GTPase Ran mediates chromatin signaling to control cortical polarity [5] and regulates meiotic spindle organization in mouse oocytes [6]. The evolutionarily conserved polarity proteins Par3 and Par6 are expressed in mouse oocytes and concentrate at the actin cap region, suggesting roles in establishing asymmetry in the oocytes [7, 8]. Moreover, the small GTPases Rac and Cdc42 likewise localize at the actin cap region and are required for oocyte polarization and spindle stabilization and positioning in 
mouse oocytes $[9,10]$.

Partner of Inscuteable (Pins) is an evolutionarily conserved polarity protein. In Drosophila neuroblasts, Pins regulates apical-basal spindle orientation and asymmetric cell division [11]. In Caenorhabditis elegans one-cell embryos, the Pins functional counterparts GPR-1/2 are essential for translating polarity cues into asymmetric spindle positioning $[11,12]$. Mammals have two Pins homologs, AGS3 (Activator of G-protein Signaling 3) and LGN (named after ten Leucine-Glycine-Asparagine tripeptides in its N-terminal region). AGS3 is tissuespecifically expressed, primarily being enriched in the brain, testis and heart, whereas LGN is ubiquitously expressed and is present in all tissues and cell lines tested [13]. AGS3 exhibits a non-homogenous distribution in the cytoplasm and is generally excluded from the nucleus [13]. AGS3 is reported to modify G $\beta \gamma$ signaling to regulate the spindle orientation of cortical progenitor cells during neurogenesis [14]. The localization and function of LGN have been examined in several cultured cell lines and some tissues. Blumer et al. [13] reported that in PC12 cells, LGN moves from the nucleus to the midbody structure separating daughter cells during the later stages of mitosis, suggesting a role in cytokinesis. However, Kaushik et al. [15] found that in WISH, PC12 and NRK cells, LGN localizes to the perinuclear region or the cytoplasm during interphase and is mainly redirected to the cortex during mitosis. They found that interfering with LGN function in cultured cell lines causes early disruption of cell cycle progression. Moreover, in MDCK cells, LGN is diffused throughout the cell in interphase, but becomes concentrated at the cell cortex and spindle poles during mitosis [16]; this finding is similar to what is observed in CHO cells [17]. At metaphase, LGN regulates mitotic spindle organization or chromosome oscillation by interacting with and inhibiting nuclear mitotic apparatus protein (NuMA), which is essential for mitotic spindle pole formation and maintenance $[16,18,19]$. LGN is asymmetrically localized in neuroepithelial progenitors and epidermal cells and regulates mammalian neurogenesis [20] and skin stratification [21], respectively.

Although a role for LGN in mitosis has been defined, its function in meiosis is unclear. In this study, we investigated the role of LGN in mouse oocytes. We examined the subcellular localization of LGN during oocyte maturation and analyzed the effects of LGN overexpression and depletion on meiotic spindle organization. Furthermore, domain dissection showed the functional separability of LGN's N-terminus and C-terminus. Our results revealed the cortical polarization of LGN during oocyte maturation and the critical role of LGN in meiotic spindle organization.

\section{Results}

\section{Expression and localization of LGN in mouse oocytes}

Mammals have two Pins homologs, AGS3 and LGN. To investigate the roles of mammalian Pins in mouse oocytes, we examined whether AGS3 and LGN were expressed. RT-PCR results indicated that LGN mRNA, but not AGS3 mRNA, was expressed in oocytes (Figure 1A). Western blot analysis demonstrated the presence of LGN protein in both GV and MII oocytes (Figure 1B). Therefore, LGN was expressed in oocytes from the GV to MII stages.

To examine the subcellular localization of LGN, we performed immunocytochemical staining on oocytes at different stages of maturation (Figure 1C). At the GV stage, LGN was distributed in the cytoplasm and at the cortex. Evidently, the cortical distribution of LGN was uniform at this point. At $1 \mathrm{~h}$ after GVBD, when chromatin was condensing and microtubules were polymerizing around, LGN congregated around the chromatin, and partially colocalized with the microtubules. When oocytes progressed into MI, the LGN signal was concentrated at the spindle apparatus, suggesting that it might play a role in spindle organization. Interestingly, the cortical distribution of LGN became polarized and less concentrated at the actin cap region. For MII-arrested oocytes, the localization pattern of LGN was identical to that of MI oocytes.

To confirm the localization dynamics of LGN, we microinjected mRNA encoding green fluorescent protein (GFP)-tagged LGN (LGN-GFP) into GV-arrested oocytes and detected the fluorescent signal during oocyte maturation. We found that LGN-GFP also localized to the cortex and became polarized during maturation; localization of LGN-GFP on the spindle apparatus was detected simultaneously (Figure 1D). Both N-terminal and C-terminal GFP tag-fused LGN had localization dynamics similar to endogenous LGN. These results indicate that LGN is cortically polarized during mouse oocyte maturation and might play a role in meiotic spindle organization.

\section{Overexpression of LGN induces meiotic spindle organi- zation defects}

To explore the function of LGN in mouse oocytes, we overexpressed LGN by injecting synthetic mRNA into GV-arrested oocytes. After culture in milrinonesupplemented medium for 6-8 h, the GV-arrested oocytes were released for spontaneous maturation. Western blot analysis showed that the amount of LGN was increased by about fourfold (Supplementary information, Figure $\mathrm{S} 1$ ), and the effect of LGN overexpression in the oocytes 


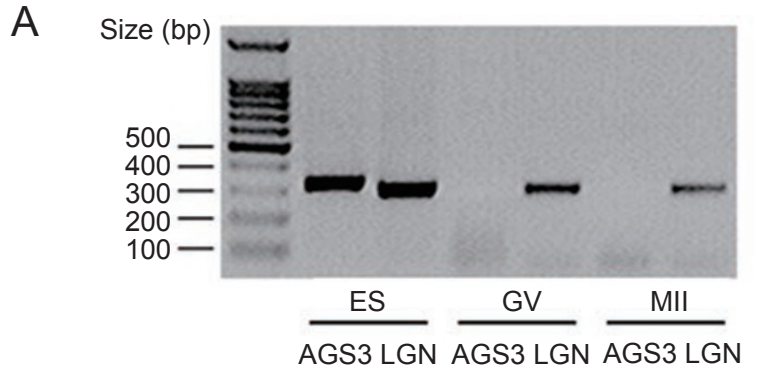

C
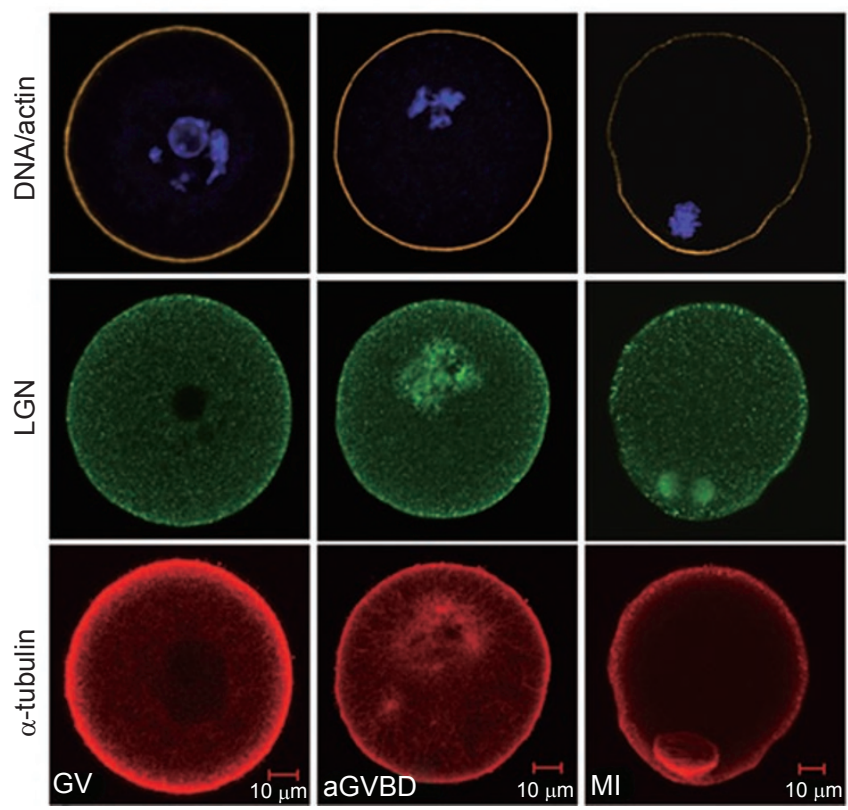
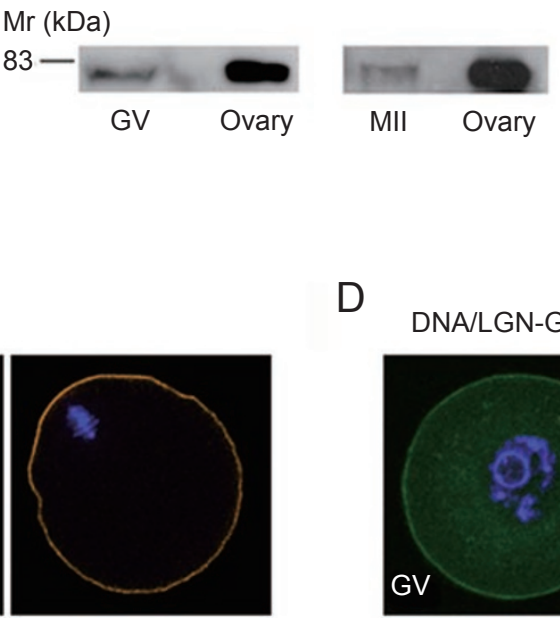

LGN

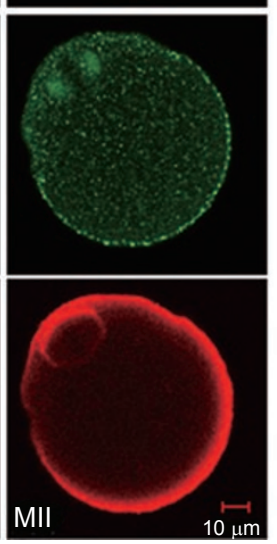

D

DNA/LGN-GFP
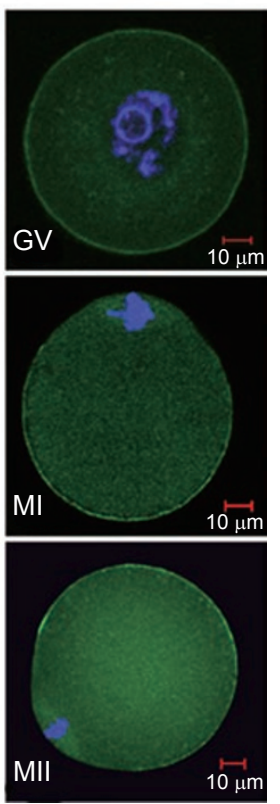

Figure 1 Expression and localization of LGN in mouse oocytes. (A) Specific primer pairs were used to perform RT-PCR on mouse embryonic stem (ES) cells, GV and MII oocytes. (B) Total proteins from mouse ovary, GV and MII oocytes were separated and blotted with antibody against mouse LGN. (C) GV, aGVBD (1 h after GVBD) MI, MIl oocytes stained with DAPI (blue), rhodamine phalloidin (orange), a-tubulin (red) and LGN antibody (green). (D) GV, MI, MIl oocytes expressing LGN-GFP, stained with DAPI.

was examined by immunocytochemical staining. In $86 \%$ (274/320) of LGN-overexpressing oocytes, the spindle was stretched and was longer than the control spindle. Furthermore, the spindle microtubules were tightly bundled and the spindle poles were extremely focused. In contrast to the typical meiotic spindle, which has less focused poles than the mitotic spindle and hence is barrelshaped, the spindles of these oocytes were compressed into a diamond shape (Figure 2A). To quantify these abnormalities, we measured the spindle length and spindle pole width as depicted in the schematic (Figure 2B). Statistical analysis showed that after LGN overexpression, spindle length was increased by an average of 32\% (Figure $2 \mathrm{C}$ ), whereas spindle width was decreased to $41 \%$ of the normal value (Figure 2D). This result indicates that
LGN can facilitate spindle elongation and spindle pole focusing.

As previously demonstrated, overexpression of LGN greatly affects the assembly of the MI spindle. As a result $80 \%$ of the oocytes suffering spindle organization defects could not proceed to the MII stage. Due to these effects, we were unable to anatomize the functions of LGN in MII oocytes, specifically whether LGN could affect MII spindle organization. Therefore, we injected $L G N$ mRNA into MII-arrested oocytes and examined them by immunocytochemistry $6 \mathrm{~h}$ later. We found that LGN overexpression could also disturb MII spindle organization. Similar to the condition seen with the MI spindles, $97 \%$ (453/466) of MII spindles were transformed into elongated and overfocused spindles (Figure 2E), with spindle 
length increasing by an average of $86 \%$ (Figure $2 \mathrm{~F}$ ) and spindle pole width decreasing by $67 \%$ (Figure $2 \mathrm{G}$ ). These results show that LGN can also regulate meiotic spindle organization in MII oocytes.

\section{Overexpression of $L G N$ disturbs spindle pole localization of $\gamma$-tubulin}

$\gamma$-tubulin is a component of acentriolar microtubuleorganizing centers, which function similarly to centrosomes and are essential for meiotic spindle assembly in mouse oocytes [2]. In view of the evidence that LGN localizes at the meiotic spindle and that LGN overexpression leads to spindle elongation and spindle pole overfocusing, we examined whether the localization of $\gamma$-tubulin was influenced by LGN overexpression.

As shown in Figure 3A, $\gamma$-tubulin in control oocytes was localized to the spindle pole, marking the end of spindle microtubules. Not surprisingly, after LGN overexpression $\gamma$-tubulin condensed to little rings or spots, consistent with overfocused spindle poles (Figure 3A).
However, in $84 \%$ of LGN-overexpressing oocytes, the $\gamma$-tubulin rings or spots were loosely attached (Figure 3C) to or completely detached from the spindle poles (Figure 3D). This evidence showed that the spindle polelocalized $\gamma$-tubulin was displaced owing to excessive LGN. Similarly, in MII-arrested oocytes, LGN overexpression caused $\gamma$-tubulin to condense into little rings or spots, with nearly all of them being detached from the spindle poles (Figure 3B). These results indicate that after LGN overexpression, the increasing of spindle length and overfocusing of spindle poles were accompanied by condensation and detachment of spindle pole $\gamma$-tubulin.

\section{Localization and function of $L G N N$-terminal and $C$ -} terminal regions

Like other Pins homologs, LGN contains seven tetratricopeptide repeat (TPR) motifs in its amino-terminal region and four Gai/o-Loco (GoLoco) motifs in its carboxyl-terminal region [22]. To understand the functions of these two distinct regions, we dissected LGN
A

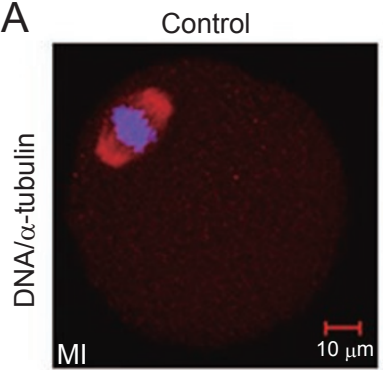

E

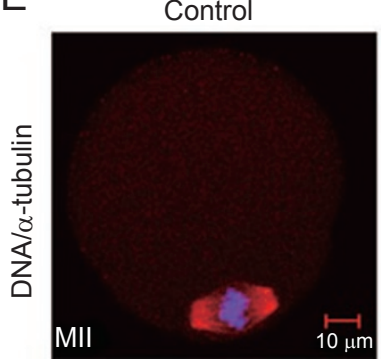

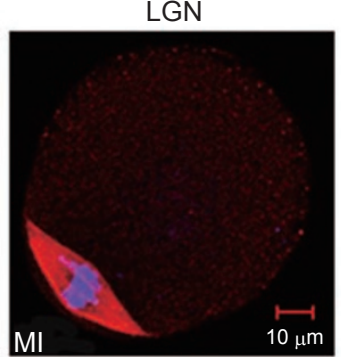

\section{C}
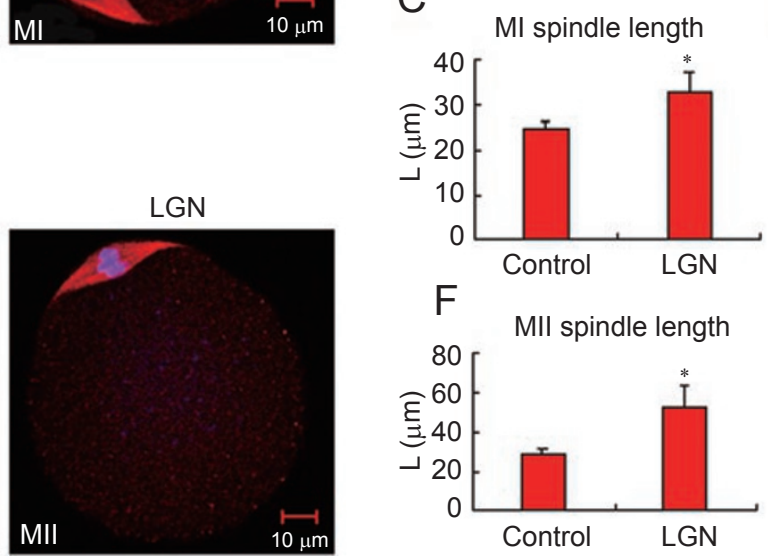

B

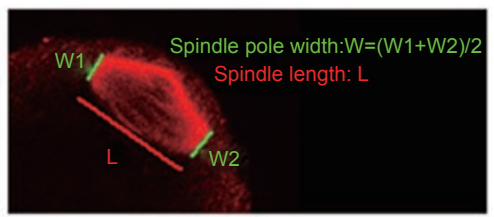

D MI spindle pole width

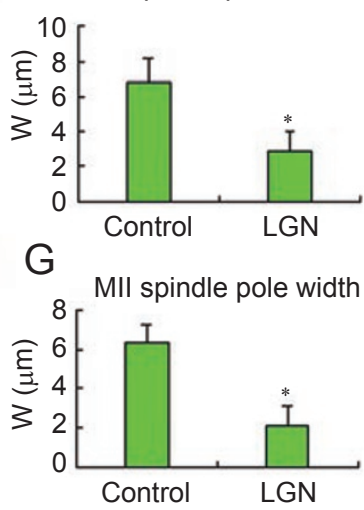

Figure 2 Overexpression of LGN induces meiotic spindle organization defects. (A) GV oocytes were injected with GFP (control) or LGN mRNA, allowed to mature to MI stage, and then stained with DAPI (blue) and $\alpha$-tubulin (red). (B) Diagram showing the measurement of spindle length and spindle pole width. (C and D) Statistics of MI spindle length and spindle pole width. Mean spindle length and spindle pole width of GFP (control) and LGN mRNA-injected oocytes are shown with errors bars (mean \pm s.d., standard deviation). A statistically significant difference was seen between GFP mRNA-injected (control) oocytes $(n=$ 26) and LGN mRNA-injected oocytes $(n=20)$. ${ }^{*} P<0.0001, t$-test. (E) MIl oocytes were injected with GFP (control) or $L G N$ mRNA and then cultured for $6 \mathrm{~h}$. (F and $\mathbf{G}$ ) Statistics of MIl spindle length and spindle pole width (mean \pm s.d.). A statistically significant difference was seen between GFP mRNA-injected (control) oocytes $(n=14)$ and LGN mRNA-injected oocytes $(n$ $=14) . * P<0.0001$, $t$-test. 

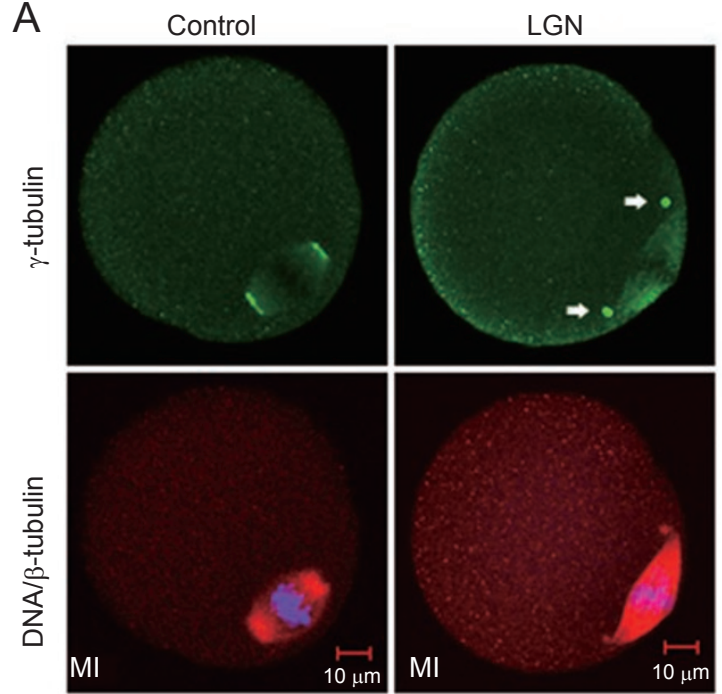

C
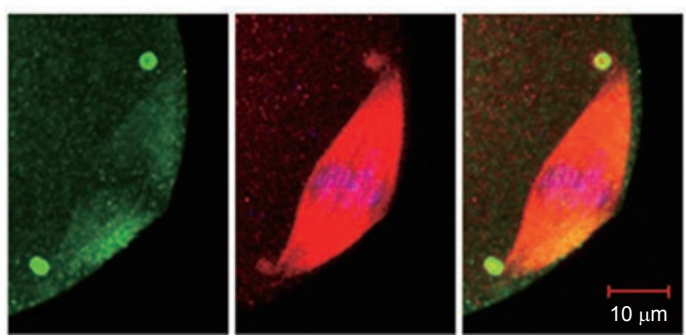

B
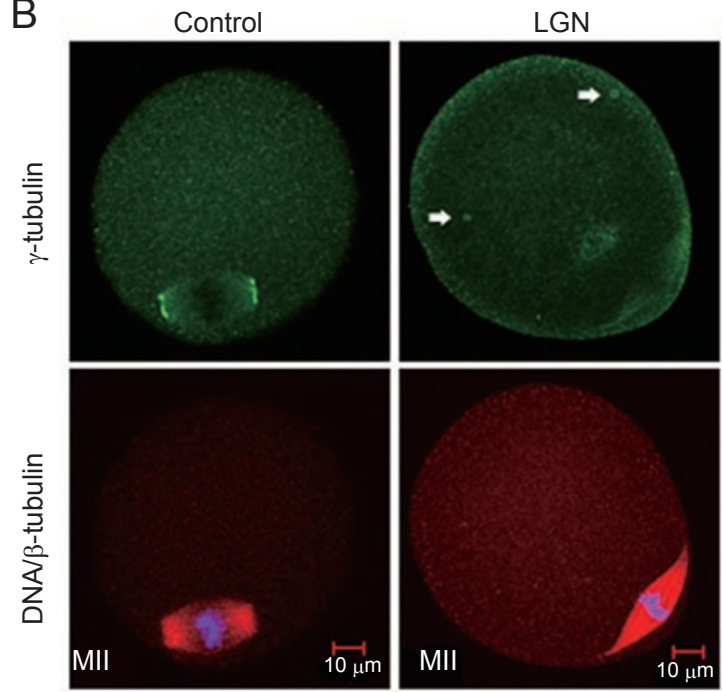

D
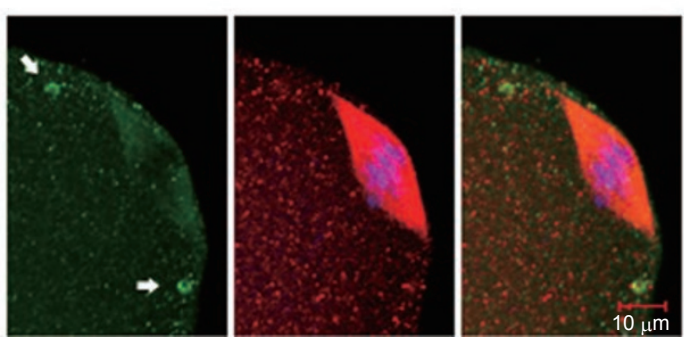

Figure 3 Overexpression of LGN disrupts spindle pole localization of $\gamma$-tubulin. (A) GV oocytes were injected with water (control) or LGN mRNA, allowed to mature to MI stage and then stained with DAPI (blue), $\beta$-tubulin (red) and $\gamma$-tubulin (green). (B) MII oocytes were injected with water (control) or LGN mRNA and then cultured for $6 \mathrm{~h}$ before staining. (C) Magnification of the affected spindle after LGN overexpression in (A), showing loosely attached $\gamma$-tubulin rings or spots. (D) Magnification of an affected spindle after LGN overexpression, showing the complete detachment of $\gamma$-tubulin. $\gamma$-tubulin rings or spots are indicated by white arrows.

and investigated the functions of its amino-terminal region (amino acid 1-373, hereafter LGN-N) and carboxylterminal region (amino acid 366-672, hereafter LGN-C) in oocytes.

LGN-N-GFP was distributed in the cytoplasm in both GV and MI oocytes. In contrast to full-length LGN, LGN-N-GFP was unable to concentrate at the cortex. However, overexpression of LGN-N-GFP in oocytes resulted in the same spindle organization defects as observed in oocytes overexpressing LGN (Figure 4A and Supplementary information, Figure S1). Excessive LGN$\mathrm{N}-\mathrm{GFP}$ elongated the spindle and overfocused the spindle poles (Figure 4C and 4D). These results indicate that the N-terminus of LGN possesses the capability of fulllength LGN to regulate meiotic spindle organization, but lacks the ability to localize at the cortex.

On the contrary, LGN-C-GFP was capable of concentrating at the cortex. At the GV stage, LGN-C-GFP local- ized evenly at the cortex, whereas at the MI stage cortical LGN-C-GFP became polarized and was eliminated from the actin cap region, similar to the dynamics of cortical LGN (Figure 4B). On the other hand, LGN-C-GFP did not significantly affect either spindle length or spindle pole width (Figure 4C and 4D), despite that its amount was 6-7 times more than that of endogenous LGN (Supplementary information, Figure S1). These results indicate that the C-terminal region of LGN is predominantly responsible for cortical localization and polarization.

\section{Downregulation of LGN by RNA interference disrupts} meiotic spindle organization

To assess the effect of loss-of-function of LGN in mouse oocytes, we used RNA interference (RNAi) to reduce the expression level of endogenous LGN. Long double-stranded RNA (dsRNA)-mediated sequencespecific RNA degradation has proved to be an effec- 
tive approach in mouse oocytes $[23,24]$. Therefore, we injected long $L G N$ dsRNA into GV-intact oocytes. The oocytes were then cultured for $40 \mathrm{~h}$ in milrinonesupplemented medium to inhibit maturation and to allow time for RNAi-mediated targeting of $L G N$ mRNA. The targeting efficiency of RNAi was assayed by quantitative real-time PCR. As shown in Figure 5A, the $L G N$ mRNA level was significantly decreased to less than $7 \%$ of the control level, in which GFP dsRNA was injected. Western blot analysis showed that the expression of LGN protein was reduced by about $50 \%$ after injection of $L G N$ dsRNA (Figure 5B), indicating that the LGN protein was relatively stable.

To observe the functional effects of LGN downregulation, the oocytes were transferred to milrinone-free medium to mature for $20 \mathrm{~h}$ and then examined by immunofluorescence. As shown in Figure 5C, meiotic spindle organization was not affected in 94\% (148/157) of control oocytes injected with GFP dsRNA. On the contrary, in $L G N$ dsRNA-injected oocytes, 39\% (67/174) of mei-

\section{A}
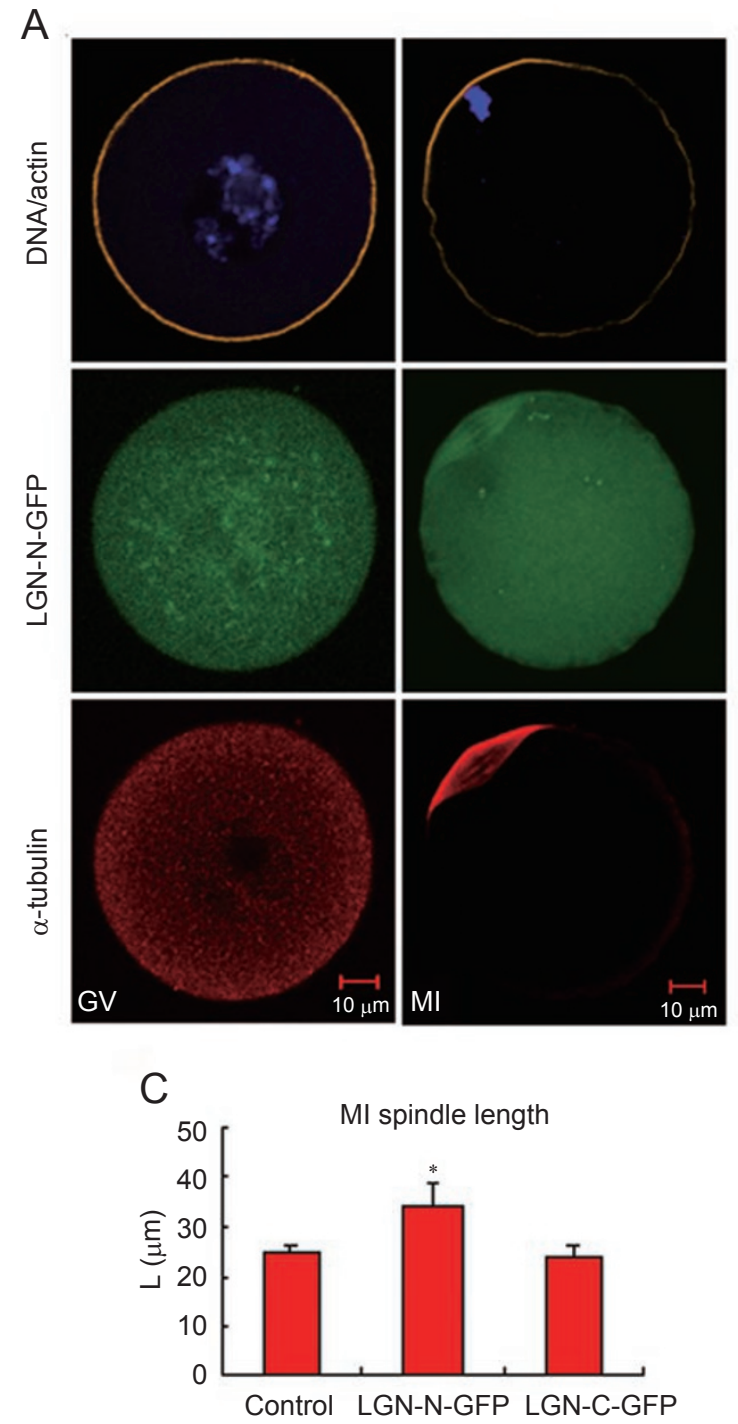
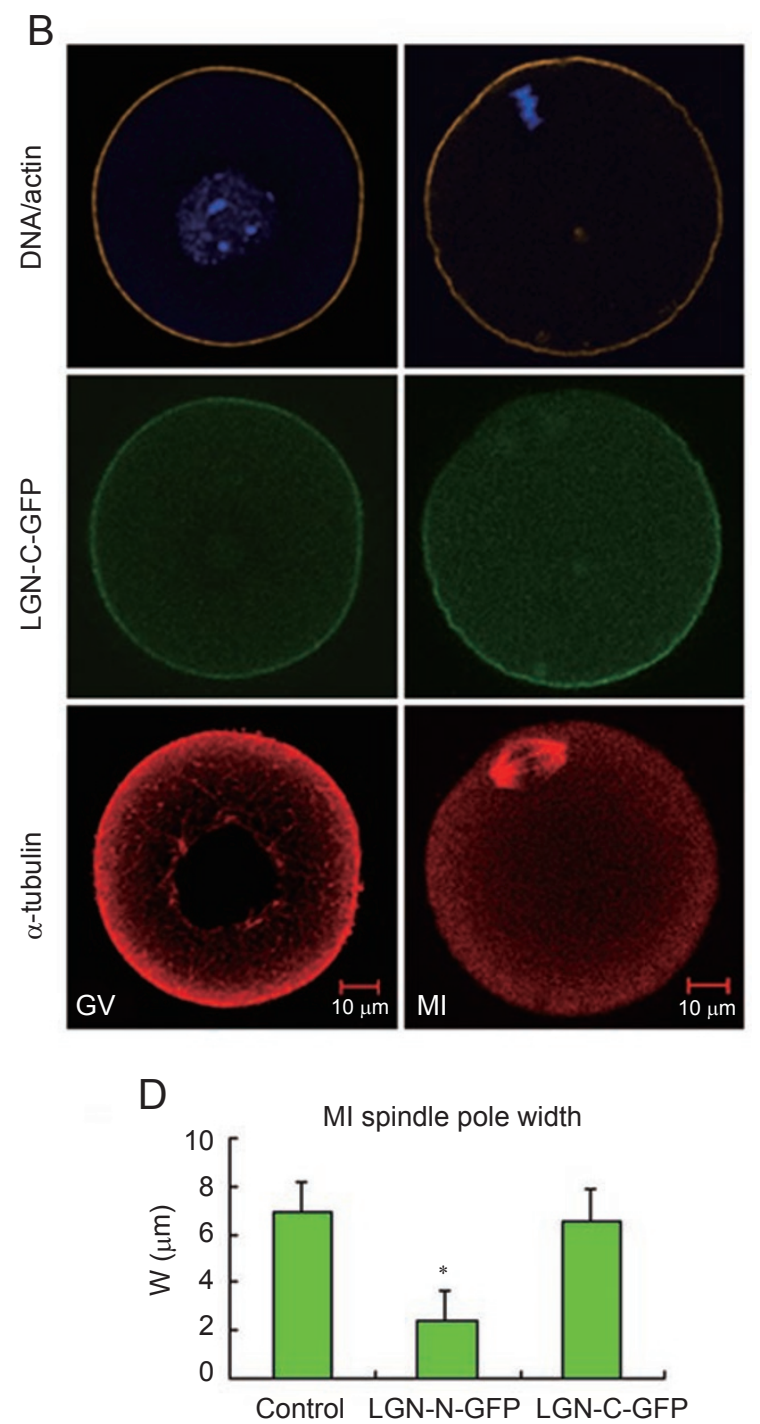

Figure 4 Functional separation of LGN N-terminus and C-terminus. GV oocytes were injected with mRNA of LGN-N-GFP or LGN-N-GFP, inhibited for several hours, and then collected or matured to MI stage and stained with DAPI (blue), rhodamine phalloidin (orange) and a-tubulin (red). (A) GV and MI oocytes expressing LGN-N-GFP. (B) GV and MI oocytes expressing LGN-C-GFP. (C and D) Quantification of LGN-N-GFP and LGN-C-GFP effects on MI spindle organization. Spindle length and spindle pole width are shown in the form of mean \pm s.d. A statistically significant difference was seen between GFP mRNAinjected (control) oocytes $(n=26)$ and LGN-N-GFP mRNA-injected oocytes $(n=25)$. However, LGN-C-GFP $(n=22)$ did not induce a significant $(P>0.05)$ effect. ${ }^{*} P<0.0001$, $t$-test. 
A

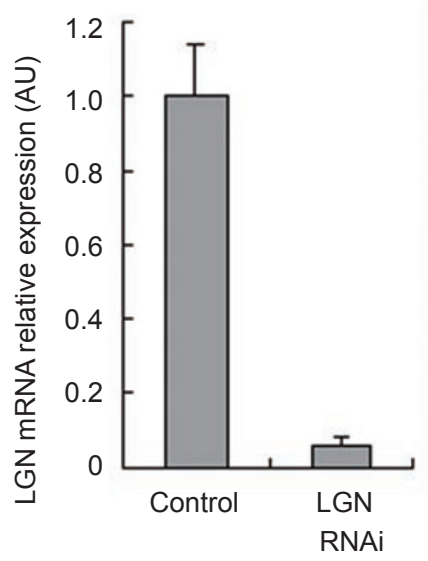

B

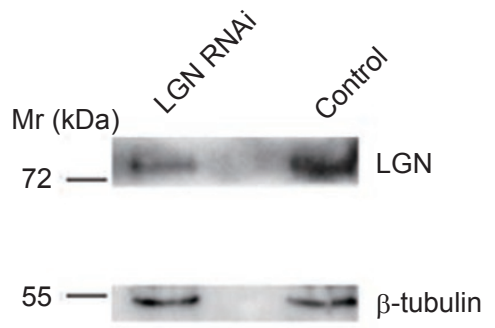

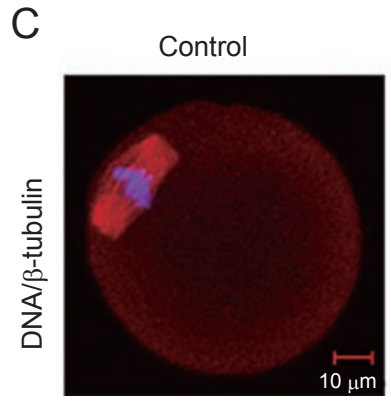

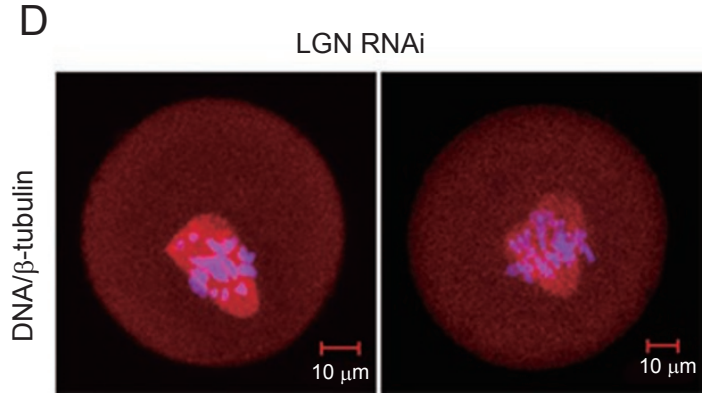

Figure 5 Depletion of LGN by RNA interference disrupts meiotic spindle organization. GV oocytes injected with LGN dsRNA (LGN RNAi) or with GFP dsRNA as a control were cultured for $40 \mathrm{~h}$ in milrinone-supplemented medium and then collected for RNAi efficiency analysis or released to mature for $20 \mathrm{~h}$. (A) Total RNA of GV-arrested oocytes (60 oocytes each sample) was isolated and used for real-time RT-PCR to quantify the level of $L G N$ mRNA. The decrease in the amount of LGN mRNA was scaled using relative arbitrary units (mean \pm s.d). Gapdh was used as an endogenous control to normalize the relative expression level. (B) The LGN protein level of GV-arrested oocytes (400 oocytes per lane) was analyzed by western blot. The same membrane was blotted with an antibody against $\beta$-tubulin as a loading control. (C and D) GFP dsRNA-injected (control) or LGN dsRNA-injected oocytes were matured for $20 \mathrm{~h}$ and stained with DAPI (blue) and $\beta$-tubulin (red).

otic spindles suffered from assembly defects. The normal barrel-shaped spindles were deformed, and chromosomes were not able to congress together and align at the equatorial plate in the $L G N$ dsRNA-injected oocytes (Figure $5 \mathrm{D})$. These results indicate that $\mathrm{LGN}$ is required for meiotic spindle assembly and chromosome alignment.

\section{Discussion}

The cortex of mouse oocytes has no apparent polarity at the GV stage, but undergoes local reorganization and polarization during meiotic spindle migration to the cortex $[1,3,4]$. In this study, we found that cortical LGN was also polarized during this process. Immunofluorescence and GFP-fused protein tracing together indicated that cortical LGN became less concentrated at the actin cap region. Domain dissection analysis showed that the C-terminus of LGN was responsible for its cortical local- ization and polarization, consistent with the findings that LGN C-terminus could localize to the cortex in mammalian cell lines [15-17]. The LGN C-terminus consists of four GoLoco motifs, which are capable of binding to heterotrimeric $\mathrm{G}$ protein $\alpha$-subunits and functioning as guanine nucleotide dissociation inhibitors [22]. In mammalian cell lines, $\mathrm{G} \alpha$ subunits of heterotrimeric $\mathrm{G}$ proteins have been shown to be required for LGN cortical localization [15]. In mouse oocytes, LGN cortical localization may also be mediated by interaction with the cortical heterotrimeric $\mathrm{G}$ protein $\alpha$-subunits. Interestingly, the polarization pattern of cortical LGN is distinct from several identified polarity proteins (such as conserved polarity mediators Par3 and Par6, and the small GTPases Rac and Cdc42), which are concentrated at the actin cap region [7-10], whereas it is similar to those of cortical granules and microvilli $[1,4]$. The specific function of cortical polarized LGN has not been elucidated, 
but our results indicate that cortical polarization not only requires the accumulation of polarity factors in the actin cap region, but also involves the elimination of specific proteins from such region such as LGN.

In Drosophila neuroblasts and C. elegans one-cell embryos, Pins or its counterparts regulate spindle orientation and positioning and thus asymmetric cell division, but do not affect spindle organization [11]. On the contrary, in cultured mammalian cells, asymmetric localization of LGN is not apparent [13, 15-17], probably because the cells lose their orientation features when cultured in vitro. However, LGN has a significant effect on mitotic spindle organization [19]. In mouse oocytes, besides localizing and polarizing at the cortex, LGN also concentrates at the spindle apparatus. Consistent with this evidence, LGN overexpression led to meiotic spindle organization defects, which included problems of elongated spindle and overfocused spindle poles.

The dynamic balance between microtubule assembly at the plus-ends and disassembly at the minus-ends near spindle poles is involved in controlling spindle length. In vitro spindle assembly assays using Xenopus laevis egg extracts indicate that dynein/dynactin and NuMA regulate spindle length by targeting the microtubuledepolymerizing factor Kif2a to spindles poles. Inhibition of Kif2a, dynactin or NuMA results in increased spindle microtubule length [25]. Previously, researchers have reported that LGN regulates mitotic spindle organization in somatic cells by interacting with and inhibiting the microtubule binding and stabilizing activity of NuMA $[16,18]$. In mouse oocytes, instead of concentrating at the spindle poles like $\gamma$-tubulin, NuMA is localized along spindle microtubules [26], similar to the spindle localization of LGN. Therefore, excessive LGN might inhibit NuMA and consequently disturb the targeting of Kif2a and/or other microtubule depolymerizing factors to the spindle poles and ultimately result in elongated meiotic spindles in mouse oocytes. Spindle length might also be influenced by modulating the pulling forces of the astral microtubules on the spindle poles. In C. elegans one-cell embryos, polarity cues result in an unequally increased pulling force on the posterior spindle, accompanied by vigorous rocking and elongation of the spindle. The GoLoco-containing proteins GPR-1 and GPR-2, as well as the cortical G $\alpha$ subunits GOA-1 and GPA-16 are essential for generation of proper pulling forces and thus asymmetric spindle positioning [12]. In MDCK cells, LGN and Gai expression induced pronounced chromosome oscillation during mitosis, which is likely a result of unbalanced pulling forces acting on the mitotic apparatus through the aster microtubules [16]. In mouse oocytes, the meiotic spindle assembles without centrosomes and is traditionally considered to lack aster microtubules. However, recent high-resolution confocal imaging in live maturing mouse oocytes indicated that the bipolar spindle surprisingly contained astral-like microtubules that probed the oocyte cortex and may therefore participate in spindle bipolarization and positioning [2]. This suggests that the spindle elongation observed in LGNoverexpressing oocytes may alternatively stem from the increased pulling forces acting on the spindle poles. LGN and Gai may be involved in the relay of information between the spindle and the cortex. A third possibility for the spindle elongation might be a combined effect of both increased spindle microtubule polymerization and enhanced pulling forces on the spindle poles, as suggested in the work of Blumer et al. [17], in which overexpression of LGN and Gai3 altered the position of the spindle poles closer to the cell cortex in $\mathrm{CHO}$ cells.

Previous experiments have defined at least three distinct motor activities that are involved in focusing microtubules at spindle poles in vertebrate cells: the minus end-directed activities of cytoplasmic dynein and the ncd (Kar3) family of kinesin-related proteins, and the plus end-directed activity of the bimC (Eg5) family of kinesin-related proteins [27-29]. Experiments using extracts prepared from synchronized cultured cells suggest that these motor activities exist in a complex balance such that the focusing of microtubule minus ends at spindle poles is favored under any experimental condition where minus end-directed motor activity predominates [30, 31]. In LGN-overexpressing oocytes, although the activity of NuMA and its associated dynein and dynactin might be inhibited, minus end-directed motor activity was still likely to be dominant, such that the driving force for focusing microtubule minus ends into spindle poles was sustained. With the elongation of spindle microtubules, the spindle pole focusing force was somehow enhanced simultaneously, leading to overfocused spindle poles (as indicated by the condensed $\gamma$-tubulin rings or spots). Intriguingly, these condensed $\gamma$-tubulin rings or spots were observed to be loosely attached or completely detached from the spindle poles, and appeared to be nonfunctioning. In cultured Drosophila S2 cells, centrosome detachment has been observed in dynein/dynactindepleted cells, ncd-depleted cells and Asp-depleted cells $[29,32,33]$. In our experiments, $\gamma$-tubulin dissociation from the spindle poles might stem from the inhibition of the dynein/dynactin/NuMA trimolecular complex by LGN overexpression. In view of the analysis that the centrosome is dispensable for spindle pole function in somatic cells once the spindle pole is organized [27], we speculate that in our experiments the dissociation of $\gamma$-tubulin occurred after the organization of the meiotic 
spindle poles.

Domain dissection analysis showed that the N-terminus of LGN possessed the capability of full-length LGN to regulate meiotic spindle organization. Overexpression of LGN-N-GFP likewise induced meiotic spindle elongation and caused overfocusing of spindle poles. The LGN N-terminus contains seven TPR motifs, which are responsible for protein-protein interactions. The N-terminus of LGN directly interacts with NuMA and localizes exclusively to the spindle poles in MDCK cells $[16,19]$. In spindle assembly assays using $X$. laevis egg extracts, addition of the LGN-N protein induced the elongation of spindle microtubules, probably by inhibiting the interaction of NuMA with microtubules [25]. In mouse oocytes, LGN-N-GFP localized to the spindle apparatus, but was not restricted to the spindle poles. This localization pattern is similar to that of NuMA, which is localized along the meiotic spindle microtubules in mouse oocytes [26]. We speculate that the localization difference between LGN (and LGN-N) on mitotic spindles in MDCK cells and LGN (and LGN-N) on meiotic spindles in mouse oocytes may stem from the different localization of NuMA. In mouse oocytes, LGN-N-GFP could induce meiotic spindle organization defects like full-length LGN, probably by direct interaction with and inhibition of NuMA. Because overexpression of LGN-C-GFP has no effect on meiotic spindle organization, the spindle-regulating activity of LGN may be restricted to its N-terminus, as observed in cell lines [17, 19]. Due to the localization of LGN-N-GFP and its expression level (about five times more than endogenous LGN), it could not be excluded that most endogenous LGN on the spindle might be competitively replaced by LGN-N-GFP, given that the $\mathrm{N}$-terminus of LGN could fulfill or substitute for the required functions and interactions of full-length LGN on the spindle. However, this replacement was not equivalent to LGN reduction or depletion because LGN-N-GFP functions similarly to LGN at the spindle and endogenous LGN is still functional whether it is localized to the spindle or not, making the overexpression effect of LGNN-GFP similar to that of LGN.

Double-stranded RNA-mediated mRNA degradation is an effective approach to knock down a gene in mouse oocytes without inducing an apoptotic response [23, 24, 34]. Using this technique, we reduced the maternally stored $L G N$ mRNA by more than $90 \%$ by $40 \mathrm{~h}$. Although LGN protein has a low turnover rate and was only reduced by about $50 \%$, dsRNA-mediated downregulation of LGN revealed its roles in meiotic spindle organization. After LGN depletion, normal meiotic spindles could not form and chromosomes were unable to congress together and align at the equatorial plate. It was difficult to direct- ly compare these defects to the effects of LGN overexpression, but they were consistent with the localization of LGN during maturation, wherein it congregated near the chromatin just after GVBD and localized at the spindle apparatus at metaphase. On the other hand, LGN protein level was half-sustained, precluding the observation of complete loss-of-function phenotypes of LGN. More robust depletion of LGN using other approaches (such as a conditional knock-out) should help define more precisely the role of LGN in mouse oocytes.

\section{Materials and Methods}

All studies adhered to procedures consistent with the National Institute of Biological Sciences Guide for the care and use of laboratory animals. All inorganic and organic compounds were purchased from Sigma Chemical Company (St Louis, MO, USA) unless otherwise stated.

\section{Oocytes collection and culture}

Oocytes were collected from female C57BL/6 or BDF 1 (C57BL/6×DBA2) mice. Fully grown GV stage oocytes surrounded by several layers of cumulus cells were collected in M2 medium by puncturing the follicles of ovaries and then pipetting to remove cumulus cells. GV oocytes were cultured and matured in Waymouth's MB 752/1 medium (GIBCO) with 10\% fetal bovine serum (Hyclone) and $0.23 \mathrm{mM}$ sodium pyruvate or in $\mathrm{CZB}$ medium in a $5 \% \mathrm{CO}_{2}, 37^{\circ} \mathrm{C}$ incubator. To inhibit GVBD, oocytes were manipulated and cultured in mediums supplemented with milrinone at a concentration of $2.5 \mu \mathrm{M}$. MII oocytes were obtained from the oviducts of females primed with pregnant mare serum gonadotrophin and then human chorionic gonadotropin. After cumulus cells were dispersed with $0.3 \%$ hyaluronidase, MII oocytes were cultured in CZB medium.

\section{RNA isolation, RT-PCR and plasmids construction}

Total RNAs from GV and MII oocytes were isolated using the PicoPure RNA Isolation Kit (Arcturus) according to the manufacturer's instructions. cDNAs were synthesized with the RETROscript (Ambion Inc.) kit and then used as templates for PCR amplification. Real-time PCR was performed using SYBR Premix EX Taq (TaKaRa) on a Mastercycler ep realplex (Eppendorf). The Gapdh gene was detected in parallel and used as the internal control. Primer pairs were used for AGS3: CTG GAC AGT GCT GAT GTA AGG G and TGT TGA GGG TGA TGC GTA GC; for $L G N$ : CAA AGG AGT TCG GAG ATA AGG and TGT GTA TGC GTT TCC TAA GC; for Gapdh: TGG CAA AGT GGA GAT TGT TGC $\mathrm{C}$ and AAG ATG GTG ATG GGC TTC CCG. To construct plasmids for in vitro transcription, DNA fragments of $L G N$ were amplified from cDNA of C57BL/6 GV oocytes. DNA fragments were inserted into a modified pBluescriptRN3 vector (a generous gift from JB Gurdon, Wellcome Trust/Cancer Research UK Institute). Fused proteins with GFP tag at the $\mathrm{N}$-terminus or C-terminus were constructed.

\section{In vitro $m R N A$ and $d s R N A$ production and microinjection}

Capped mRNAs were transcribed from linearized plasmids with the mMessage mMachine kit (Ambion Inc.), purified with the 
RNeasy MinElute Cleanup Kit (QIAGEN) and eluted in RNasefree water. For dsRNA production, cDNA inserts were amplified by PCR using primers to attach $\mathrm{T} 7$ promoter recognition sites to both the $5^{\prime}$ and the $3^{\prime}$ ends. Complementary dsRNA was generated from the PCR product using the T7 RiboMAX Express Large Scale RNA Production System. The following primers were used for $L G N$ : TAA TAC GAC TCA CTA TAG GGA GAC AGT GAA ATT CTC GCT AAA C and TAA TAC GAC TCA CTA TAG GGA GAT TCC TTT AGT CCA AAC TCA C; for control GFP: TAA TAC GAC TCA CTA TAG GGA GAG GGG TGG TGC CCA TCC TG and TAA TAC GAC TCA CTA TAG GGA GAC GGC GGT CAC GAA CTC CAG. The length of dsRNA product was 715 bp for $L G N$ and 652 bp for GFP. Both mRNA and dsRNA were aliquoted and stored at $-80^{\circ} \mathrm{C}$ until use. Synthesized mRNAs or dsRNAs were microinjected into the cytoplasm of GV-arrested oocytes via a FemtoJet microinjector (Eppendorf), or MII oocytes via Piezo-Micromanipulator. Microinjection was performed in M2 or HCZB medium. Milrinone was supplemented as needed.

\section{Immunocytochemistry and confocal microscopy}

Oocytes were briefly exposed to acidic Tyrode's solution to remove the zona pellucida and fixed in 4\% paraformaldehyde in PBS for at least $1 \mathrm{~h}$ at room temperature and then permeabilized with $0.25 \%$ Triton X-100 in PBS for 15 min and blocked with $10 \%$ normal goat serum for $1 \mathrm{~h}$. The oocytes were incubated with primary antibodies for $2 \mathrm{~h}$ at $37^{\circ} \mathrm{C}$ or overnight at room temperature, washed three times in PBS and then incubated with secondary antibodies for $2 \mathrm{~h}$ at $37^{\circ} \mathrm{C}$ in the dark. The primary antibodies used included monoclonal anti- $\alpha$-tubulin, polyclonal anti- $\beta$-tubulin (Santa Cruz), monoclonal anti- $\gamma$-tubulin and polyclonal antiGPSM2 (ProteinTech Group, Inc.). The secondary antibodies used included Alexa Fluor 594 Goat Anti-Mouse, Alexa Fluor 594 Goat Anti-Rabbit, Alexa Fluor 488 Goat Anti-Mouse and Alexa Fluor 488 Goat Anti-Rabbit (Molecular Probes). For microfilaments staining, rhodamine phalloidin (Molecular Probes) was used along with secondary antibodies. After being washed, oocytes were mounted in Prolong Gold antifade reagent (Molecular Probes) with $500 \mathrm{ng} / \mathrm{ml} \mathrm{4',6-diamidino-2-phenylindole} \mathrm{(DAPI)} \mathrm{(Molecular}$ Probes).

Confocal images were acquired with a Zeiss LSM 510 Meta microscope. Section projection and measurements of spindle length and spindle pole width were done using an LSM Image Browser. Numerical computation and chart construction were performed in Excel. Images and charts were organized using Adobe Photoshop software.

\section{Western blot}

Collected cumulus-free GV and MII oocytes were lysed by boiling in SDS-PAGE loading buffer at $100{ }^{\circ} \mathrm{C}$ for $5 \mathrm{~min}$. Total oocyte proteins were separated by $10 \%$ polyacrylamide gels and subsequently electrotransferred onto Hybond-P PVDF membranes (Amersham). Membranes were blocked with 5\% non-fat dried milk in TBS containing $0.05 \%(\mathrm{v} / \mathrm{v})$ Tween 20 for $2 \mathrm{~h}$ at room temperature, incubated with diluted rabbit antibody against mouse LGN (kindly provided by Xiaohang Yang, Institute of Molecular and Cell Biology, Singapore) and polyclonal anti-GPSM2 (ProteinTech Group, Inc.) overnight at $4{ }^{\circ} \mathrm{C}$, and incubated with HRPlabeled goat anti-rabbit antibody (Amersham) for $2 \mathrm{~h}$ at room temperature. The antigens were detected using ECL Plus Western
Blotting Detection Reagents (Amersham).

\section{Acknowledgments}

We thank JB Gurdon for kindly supplying the pBluescriptRN3 vector, Xiaohang Yang for generously sharing the LGN antibody, and Yu Zhang and Zhaohui Kou in our lab for technical assistance. This work was supported by National High Technology Project 863 (2005AA210930).

\section{References}

1 Maro B, Verlhac MH. Polar body formation: new rules for asymmetric divisions. Nat Cell Biol 2002; 4:E281-E283.

2 Schuh M, Ellenberg J. Self-organization of MTOCs replaces centrosome function during acentrosomal spindle assembly in live mouse oocytes. Cell 2007; 130:484-498.

3 Brunet S, Maro B. Cytoskeleton and cell cycle control during meiotic maturation of the mouse oocyte: integrating time and space. Reproduction 2005; 130:801-811.

4 Sun QY, Schatten H. Regulation of dynamic events by microfilaments during oocyte maturation and fertilization. Reproduction 2006; 131:193-205.

5 Deng M, Suraneni P, Schultz RM, Li R. The Ran GTPase mediates chromatin signaling to control cortical polarity during polar body extrusion in mouse oocytes. Dev Cell 2007; 12:301-308.

6 Dumont J, Petri S, Pellegrin F, et al. A centriole- and RanGTP-independent spindle assembly pathway in meiosis I of vertebrate oocytes. J Cell Biol 2007; 176:295-305.

7 Duncan FE, Mossa SB, Schultz RM, Williams CJ. PAR-3 defines a central subdomain of the cortical actin cap in mouse eggs. Dev Biol 2005; 280:38-47.

8 Vinot Sp, Le T, Maro B, Louvet-Vallée S. Two PAR6 proteins become asymmetrically localized during establishment of polarity in mouse oocytes. Curr Biol 2004; 14:520-525.

9 Halet G, Carrol J. Rac Activity is polarized and regulates meiotic spindle stability and anchoring in mammalian oocytes. Dev Cell 2007; 12:309-317.

10 Na J, Zernicka-Goetz M. Asymmetric positioning and organization of the meiotic spindle of mouse oocytes requires CDC42 function. Curr Biol 2006; 16:1249-1254.

11 Betschinger J, Knoblich JA. Dare to be different: asymmetric cell division in Drosophila, C. elegans and vertebrates. Curr Biol 2004; 14:R674-R685.

12 Colombo K, Grill SW, Kimple RJ, et al. Translation of polarity cues into asymmetric spindle positioning in Caenorhabditis elegans embryos. Science 2003; 300:1957-1961.

13 Blumer JB, Chandler LJ, Lanier SM. Expression analysis and subcellular distribution of the two g-protein regulators AGS3 and LGN indicate distinct functionality. J Biol Chem 2002; 277:15897-15903.

14 Sanada K, Tsai LH. G Protein $\beta \gamma$ subunits and AGS3 control spindle orientation and asymmetric cell fate of cerebral cortical progenitors. Cell 2005; 122:119-131.

15 Kaushik R, Yu F, Chia W, Yang X, Bahri S. Subcellular localization of LGN during mitosis: evidence for its cortical localization in mitotic cell culture systems and its requirement for normal cell cycle progression. Mol Biol Cell 2003; 14:3144- 
3155.

16 Du Q, Macara IG. Mammalian Pins is a conformational switch that links NuMA to heterotrimeric G proteins. Cell 2004; 119:503-516.

17 Blumer JB, Kuriyama R, Gettys TW, Laniera SM. The Gprotein regulatory (GPR) motif-containing Leu-Gly-Asnenriched protein (LGN) and Gia3 influence cortical positioning of the mitotic spindle poles at metaphase in symmetrically dividing mammalian cells. Eur J Cell Biol 2006; 85:12331240.

18 Du Q, Taylor L, Compton DA, Macara IG. LGN blocks the ability of NuMA to bind and stabilize microtubules: a mechanism for mitotic spindle assembly regulation. Curr Biol 2002; 12:1928-1933.

19 Du Q, Stukenberg PT, Macara IG. A mammalian partner of inscuteable binds NuMA and regulates mitotic spindle organization. Nat Cell Biol 2001; 3:1069-1075.

20 Konno D, Shioi G, Shitamukai A, et al. Neuroepithelial progenitors undergo LGN-dependent planar divisions to maintain self-renewability during mammalian neurogenesis. Nat Cell Biol 2008; 10:93-101.

21 Lechler T, Fuchs E. Asymmetric cell divisions promote stratification and differentiation of mammalian skin. Nature 2005; 437:275-280.

22 Willard FS, Kimple RJ, Siderovski DP. Return of the GDI: the GoLoco motif in cell division. Annu Rev Biochem 2004; 73:925-951.

23 Wianny F, Zernicka-Goetz M. Specific interference with gene function by double-stranded RNA in early mouse development. Nat Cell Biol 2000; 2:70-75.

24 Svoboda P, Stein P, Hayashi H, Schultz RM. Selective reduction of dormant maternal mRNAs in mouse oocytes by RNA interference. Development 2000; 127:4147-4156.

25 Gaetz J, Kapoor TM. Dynein/dynactin regulate metaphase spindle length by targeting depolymerizing activities to spindle poles. J Cell Biol 2004; 166:465-471.

26 Lee J, Miyano T, Moor RM. Spindle formation and dynamics of $\gamma$-tubulin and nuclear mitotic apparatus protein distribution during meiosis in pig and mouse oocytes. Biol Reprod 2000; 62:1184-1192.

27 Compton DA. Focusing on spindle poles. J Cell Sci 1998; 111:1477-1481.

28 Compton DA. Spindle assembly in animal cells. Annu Rev Biochem 2000; 69:95-114.

29 Goshima G, Nédélec F, Vale RD. Mechanisms for focusing mitotic spindle poles by minus end-directed motor proteins. $J$ Cell Biol 2005; 171:229-240.

30 Gaglio T, Dionne MA, Compton DA. Mitotic spindle poles are organized by structural and motor proteins in addition to centrosomes. J Cell Biol 1997; 138:1055-1066.

31 Gaglio T, Saredi A, Bingham JB, et al. Opposing motor activities are required for the organization of the mammalian mitotic spindle pole. J Cell Biol 1996; 135:399-414.

32 Morales-Mulia S, Scholey JM. Spindle pole organization in Drosophila S2 cells by dynein, abnormal spindle protein (Asp), and KLP10A. Mol Biol Cell 2005; 16:3176-3186.

33 Robinson JT, Wojcik EJ, Sanders MA, McGrail M, Hays TS. Cytoplasmic dynein is required for the nuclear attachment and migration of centrosomes during mitosis in Drosophila. J Cell Biol 1999; 146:597-608.

34 Svoboda P. Long dsRNA and silent genes strike back: RNAi in mouse oocytes and early embryos. Cytogenet Genome Res 2004; 105:422-434.

(Supplementary information is linked to the online version of the paper on the Cell Research website.) 